besser noch in Europa - endlich eine klare, konsistente Politik betrieben werden. Im Vordergrund müssen stehen:

(1) Förderung von Forschung und Entwicklung an den Universitäten sowie in den Unternehmen.

(2) Erarbeitung einer Markteinführungsstrategie, die auch die Rolle der öffentlichen Hand als wichtigen Nachfrager klärt.
(3) Einheitliche und auskömmliche Einspeisevergütungen für Strom aus regenerativen Energiequellen. Als Alternative ist die Vorgabe eines stetig wachsenden, verbindlichen Anteils von „ÖkoStrom" an der Stromversorgung denkbar.

Dann ist eine Realisierung der Ziele der EU-Kommission im Bereich der Photovoltaik sicher erreichbar.

\title{
Jugendwettbewerb für Sonnenkinder
}

\author{
G. Becker ${ }^{1}$
}

\begin{abstract}
Unser Ziel, die Jugend für eine Partnerschaft für die Zukunft zu gewinnen, haben wir im ersten Versuch sehr gut erreicht. Die Arbeiten der Jugend zeichnen sich durch Kreativität, Professionalität und ein besonders starkes Einfühlungsvermögen im Denken mit der Sonne aus. Der Youth Award wurde an fünf Gruppen in den Bereichen gemeinsame Initiative von Lehrern und Schülern, Gesamtenergiekonzept für Ladakh, elektrische Energie für Nepal, architektonische Entwürfe für eine Schule und Solarcontainer - verliehen.
\end{abstract}

Schlüsselwörter: 2. Weltkonferenz für Photovoltaik; Photovoltaikdemonstrationsprojekte der Jugend; Hybridlösungen mit Photovoltaik, Wind, Wasser und Sonnenkollektoren; Container-Kleinwasserkraftwerk; Modulare Energieversorgungssysteme; Photovoltaikcontainer

Youth Award for solar youth groups. We achieved our target to win the youth for a partnership for the future very well in the first attempt. The works of youth distinguish themselves through creativity, professionalism and a strong intuition in thinking with the sun. The Youth Award was given to five groups in the fields: common initiative of teachers and pupils, total energy concept for Ladakh, electric energy for Nepal, architectural drafts for a school and solar containers.

Keywords: 2nd world conference on photovoltaics; photovoltaic demonstration projects of the youth; hybrid solutions with photovoltaics, wind, water and thermal collectors; container-small hydro electric power plant; modular energy supply system; photovoltaic container

Prof. Schmid, der Präsident der 2. Weltkonferenz für Photovoltaik, Prof. Friedrich von der Universität Graz und Ing. Gernot Becker vom österr. Bundesverband für Photovoltaik hatten die Idee, zur Weltkonferenz auch jene Gruppe einzuladen, die aus heutiger Sicht die

\footnotetext{
' Ing. Gernot Becker, Vorstand des Bundesverbandes für Photovoltaik der Bundeswirtschaftskammer Österreichs, ATB-Antennen, Umwelt, Technik, Becker, Dörferstraße 16, A-6067 Absam.
}

größte Last der Umweltverschmutzungen zu tragen haben wird - die Jugend.

Wir als aktive Befürworter der Umwelttechniken insgesamt und der Photovoltaik im speziellen versuchen eine Trendwende hin zu einer lebenswerteren Welt und setzen dabei auf die Energiequellen, die uns von der Natur selbst gegeben werden. Dadurch wollen wir der Natur helfen, ihre eigene Kraft der Selbstreinigung zu verstärken. 
Wir stehen vor der Aufgabe, Wege für vermehrten Einsatz emissionsfreier und erneuerbarer Energien zu finden. Der Einstieg der großen Stromversorgungsunternehmen und der Ölkonzerne in die Photovoltaik zeigt, daß der aufgezeigte Weg langsam Anhänger findet, und daß die Zeit für die kommerzielle Nutzung der Photovoltaik gekommen ist. Es macht zwar nachdenklich, wenn immer nur das Zugpferd Geld und Gewinne einer Technik zum Durchbruch verhelfen, doch braucht unsere Umwelt so dringend Hilfe, daß Vorwürfe für unterlassene Hilfeleistungen heute in den Hintergrund treten müssen.

Das Ziel, unseren Kindern eine heile Welt hinterlassen zu können, ist bereits aussichtslos. Das Ziel, unseren Kindern aber nicht nur Schutt und Asche zu hinterlassen, können wir mit vereinter Anstrengung noch erreichen.

Die Jugend als Partner für die Zukunft zu gewinnen, war und ist die Idee für den Jugendwettbewerb im Rahmen der 2. Weltkonferenz für Photovoltaik in der Wiener Hofburg. Der Bundesverband für Photovoltaik (BVP) übernahm die Organisation dieses Aufrufs an die Jugend. Eine Ausschreibung der Idee folgte, und viele Gespräche mit Persönlichkeiten und aktiven Gruppen im Bereich der Umwelttechnik führten dazu, daß man an vielen Stellen Österreichs von kleineren und größeren Aktivitäten in Richtung Photovoltaik hörte. Nachträglich mußten wir feststellen, daß trotz unermüdlicher Suche viele Talente im Verborgenen blieben. Es fehlt bei vielen österreichischen Firmen einfach noch eine starke Unterstützung hin zu dieser Zukunftspartnerschaft mit der Jugend.
Der BVP übernahm auch die Unterstützung von Schüler- und Jugendgruppen durch aktive Einschulungsphasen, wenn diese von den Jugendlichen gewünscht wurde. Von einigen Jugendgruppen wurde diese Hilfe gerne auch für den Projektstart angenommen, damit die Ziele und Grundregeln für die Teilnahme am Wettbewerb analysiert werden konnten. Ganz schnell standen die Mädchen und Burschen auf eigenen Füßen, zeigten Selbstvertrauen, lehnten weitere Hilfe ab und nützten die kurze Zeit bis zur Weltkonferenz optimal.

Nicht alle Projekte erreichten auch die Ziellinie, da so manche Gruppe zu wenig Motivation durch uns Erwachsene erhielt und andere in den Vorbereitungen zur Matura einfach mit der kurzen Ausarbeitungszeit nicht zurecht kamen.

Fünf Jugendgruppen fanden sich dann zum Zeitpunkt des Aufbaus des Österreich-Events im Rahmen der 2. Weltkonferenz für Photovoltaik am Heldenplatz vor der Hofburg ein, und eine österreichische Zeitung schrieb sehr treffend:

„Kräftig sandte die Sonne zum Anfang der Weltkonferenz ihre Strahlen auf den Platz vor der Hofburg. Drinnen fand der 2. Weltkongreß zum Thema Photovoltaik statt, draußen auf der Papstbühne präsentierten sich österreichische Projekte. Ob Modelle für bestehende oder künftig mögliche Nutzung der Solarenergie - allesamt stammen sie aus den Köpfen und Händen von Kindern und Jugendlichen." (Kurier vom 13. 7. 1998)

Kräftig und aufgeregt waren auch die vielen Hände, die da letzte Arbeiten an den Modellen durchführten, um sie bestmöglich dem Publikum zu präsentieren. Die günstige Lage und die schöne Optik der Bühne, die

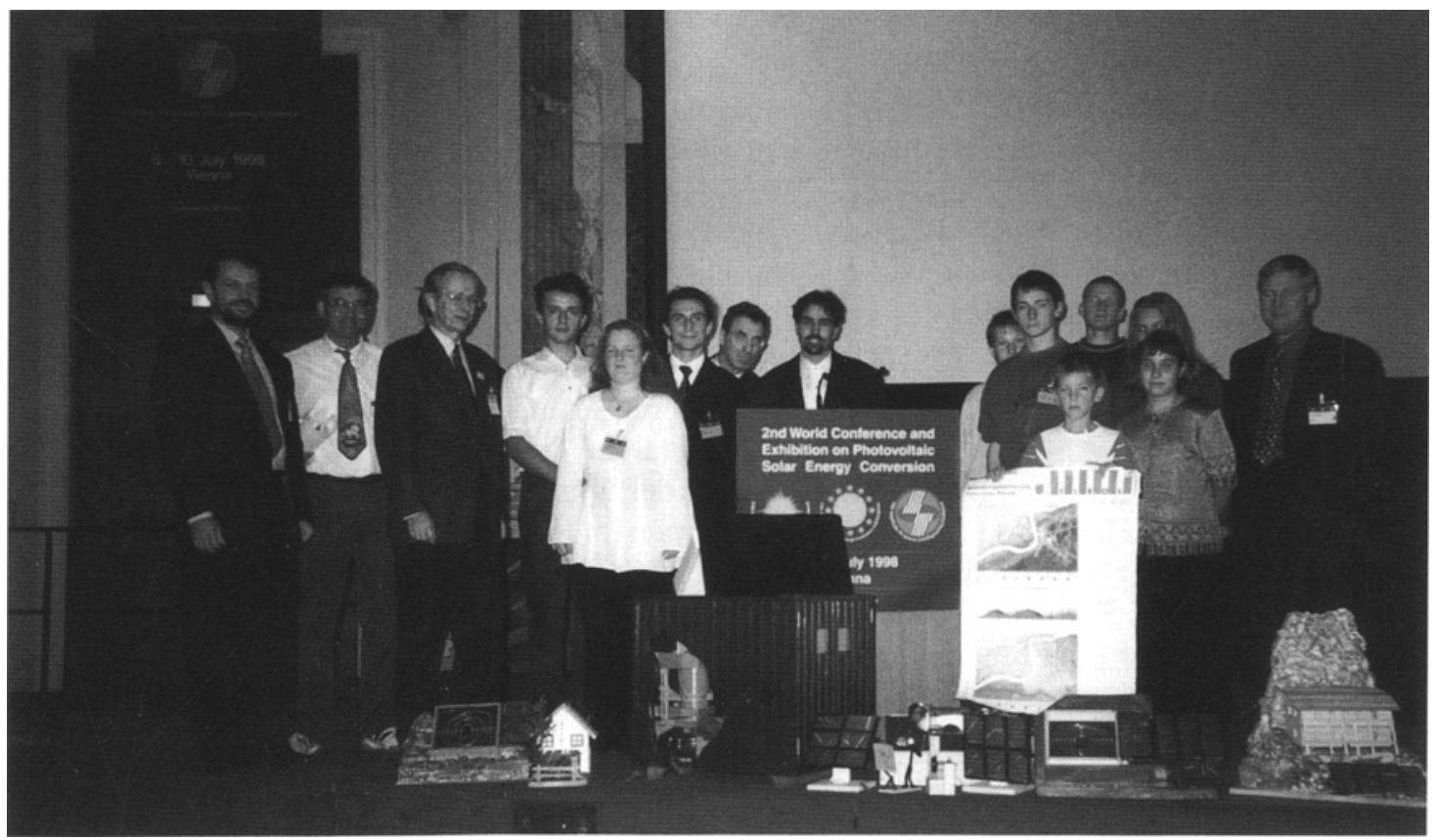

Abb. 1. Teilnehmer des Youth Award 
von Arch. Neversal für den Papstbesuch und die Feierlichkeiten zum Beginn der österreichischen EU-Präsidentschaft entworfen wurde, lockte zahlreiche Kongreßteilnehmer sowie in- und ausländische Interessierte vom vielbesuchten Heldenplatz auf die Bühne. Die Jugendlichen hatten kaum Zeit, Luft zu holen, und es war erstaunlich, wie wortgewandt die Projekterklärungen in Deutsch, Englisch und teilweise in Französisch gebracht wurden. Und sichtlich begeistert waren auch die Besucher, die bei einigen Projekten direkt Projektnutzungsmöglichkeiten andiskutierten. Ein Professor einer Universität in Chile will mit einer der Jugendgruppe über Internet in Zukunft Erfahrungen austauschen, weil er in seinem Land eine ähnliche Aufgabe zu lösen hat.

\section{Kinder im Sonnen-Dorf}

Pfunds, ein kleines Dorf im Tiroler Oberland, ist eine der ganz aktiven Klimabündnisgemeinden, die wirklich nicht von der Sonne verwöhnt werden. Ein Drittel des Dorfes kennt im Winter Wochen, in denen die Sonne den ganzen Tag nicht scheint. Und gerade diese Gemeinde kennt den Nutzen der Sonne. Die Lehrer und Kinder der Hauptschule arbeiten bereits ab dem ersten Jahrgang an Projekten, die den Kindern den Umgang und das Denken aus der Sicht der Sonne nahebringt. Und die Fröhlichkeit der Kinder zeigt, daß dieser Weg für uns alle empfehlenswert wäre.

Kathrin Apolonio, Eva-Maria Schmitzberger, Christoph Cormier, Johannes Maas und Markus Waldegger, Schüler aus der ersten und vierten Klasse der Schule, brachten ihre Modelle mit und zeigten, für welche Dinge Photovoltaik elektrische Energie liefern kann. Und sie zeigen auch, daß die Kombination von erneuerbaren Energien ein Multiplikator für den Nutzen und den Wirkungsgrad von Anlagen darstellt.

Solarzellen treiben eine Pumpe, die wiederum Wasser durch eine Schlauchspirale pumpt, das sich durch die Sonneneinstrahlung erwärmt und in einen Becher fließt, aus dem das Wasser wieder in den Kreislauf geschickt wird und noch wärmeres Wasser das Ergebnis ist. Eine vollkommen natürliche Sache wie den Kindern in einfacher Art das System der Warmwasserkollektoranlagen erklärt wird.

Eine Berghütte in einer steilen, gefährlichen Bergwand (im Modell aus Styropor) ist innen hell erleuchtet und das mit einem selbstgefertigten Photovoltaikmodul. Das Projekt zeigt, daß Licht und elektrische Energie für eine Berg- oder Schutzhütte von der Sonne direkt erzeugt werden kann und bei Speicherung der am Tage gewonnenen elektrischen Energie in einer Batterie auf stinkende Dieselaggregate verzichtet werden kann.

Ein Biotop mit einer solarbetriebenen Pumpe stellt uns den Stellenwert von Wasser in der Natur dar und mahnt uns, alle Anstrengungen zu unternehmen, da 3 wir auch in Zukunft unsere Lebensgrundlage - das reine Trinkwasser - nicht durch Dünger und Gifte gefährden.

Die Art der von Jugendlichen mitausgearbeiteten Sonnenkartierung hat die Tiroler Landesregierung übernommen und bietet interessierten Gemeinden ihre Ausarbeitung an. Die Sonnenkartierung zeigt in einem Gemeindegebiet in einzelnen Monatskarten, ob ein Wohnobjekt eine bessere oder schlechtere Lage für die Nutzung von Sonnenenergie hat.

Der Youth Award wurde den Sonnenkindern von Pfunds für die gemeinsame Initiative von Lehrern und Schülern für die erneuerbaren Energie verliehen. Die Arbeit der Jugend hat Solarenergie im Gemeindegebiet von Pfunds zu etwas Alltäglichem werden lassen (Abb. 1).

\section{Sonne für ein Kinderheim in Indien - Projekt Ladakh}

Im Norden Indiens, zwischen den höchsten Bergketten des Himalayas, in Ladakh, in der Nähe von Leh, gründete ein junger buddhistischer Mönch 1986 im Auftrag seines Lehrers ein Sozialzentrum und steckte sich sehr hohe Ziele.

In einer Fels- und Sandwüste errichtete er eine Schule und gibt begabten Kindern aus armen Verhältnissen die Möglichkeit einer umfassenden Ausbildung, die auch Ökologie und das Leben in der Einheit mit der Natur einschließt. In einem Heim gibt er 75 Waisenkindern ein neues Zuhause und versorgt in einem kleinen Altenheim alte notleidende Menschen. Ein Krankenhaus ist fast fertiggestellt und in provisorischen Räumen wurden bereits Augenoperationen durchgeführt.

Die Stromversorgung aus einem Wasserkraftwerk am nahegelegenen Indus schafft während der Sommermonate für die Gegend um die Distrikthauptstadt Leh eine Tagesdeckungsrate von nur $24,5 \%$. Im Winter kann es wegen der extremen Temperaturen (bis $-50^{\circ} \mathrm{C}$ ) nicht betrieben werden und bringt für 7 Monate/Jahr keine elektrische Energie.

Die Stromversorgung fehlt somit im Winter zur Gänze. Der Leiter des Kinderheims versichert, daß man darauf schaut, daß die Temperaturen in den Lern- und Schlafräumen der Kinder nie unter $0^{\circ} \mathrm{C}$ absinkt. Die Mädchen lernen im Kerzenlicht und nicht selten passiert es, daß sie neben der Kerze einschlafen und Brände nur durch die Aufmerksamkeit der Betreuer verhindert werden.

Vier (Ex-)HTL-Schüler hörten von Ladakh und dem Unterstützungsverein „Projekt Ladakh“, vertieften ihr Wissen über Ladakh und meinten, ,da machen wir mit". Karin Dallio hat die HTL für Hochbau und Valentin Simic die HTL für Nachrichtentechnik bereits 
abgeschlossen, während Thomas und Florian Becker noch Schüler an der HTL-Innsbruck für Elektrotechnik sind. Hinzu kam Maya Shanker eine Philosophie- und Hindi-Studentin aus Linz, die die Nähe zur Kultur in das Projekt einbrachte.

Das Projekt Ladakh der Jugendgruppe umfaßt die Ausarbeitung eines Gesamtenergiekonzepts auf Basis von Photovoltaik und Windkraftanlagen zur Erzeugung elektrischer Energie und thermischer Kollektoranlagen zur Gewinnung von. Warmwasser für die Heizung. Alle Teile wurden modular vorgesehen, damit die Realisierung des Projekts in verschiedenen Bauabschnitten, den eingehenden Spenden entsprechend, möglich ist. Die gesamte Anlage wird dann als Insel betrieben und macht sich so unabhängig vom vollkommen unzureichenden Stromnetz, das als zusätzliche Energiequelle einen Beitrag liefert.

Die Projekt-Ladakh-Jugend denkt sehr gesamtheitlich auch an die Verbesserung der Isolation der Gebäudeaußenhülle und sucht landesübliche Isoliermaterialien und Handwerker die die Ausführung machen werden.

Maya Shanker flog direkt von der Weltkonferenz nach Ladakh, um sich vor Ort ein Bild über die Verhältnisse und Möglichkeiten bezüglich Wind- und Sonnenenergie zu machen und den Menschen im Zentrum die Ergebnisse der Jugendarbeit vorzustellen. Als ernstgemeinte Unterstützung wird noch 1998 eine Meßanlage für die Aufnahme der Winddaten und der Sonneneinstrahlungwerte installiert, die bis Dezember 1998 Grundlagen für die Feinausarbeitung liefert.

Der Projekt-Ladakh-Jugendgruppe wurde der Youth Award für ihre Bemühungen um eine bessere und lebenswertere Zukunft verliehen (Abb. 2). Der Einsatz für erneuerbare Energien ist wegweisend für zukünftige Generationen ausgearbeitet. Die Gruppe hat in vorbildlicher Weise ein Gesamtenergiekonzept ausgearbeitet. Sonnen- und Windenergie liefern Strom und Wärme für Kinder und alte Menschen.

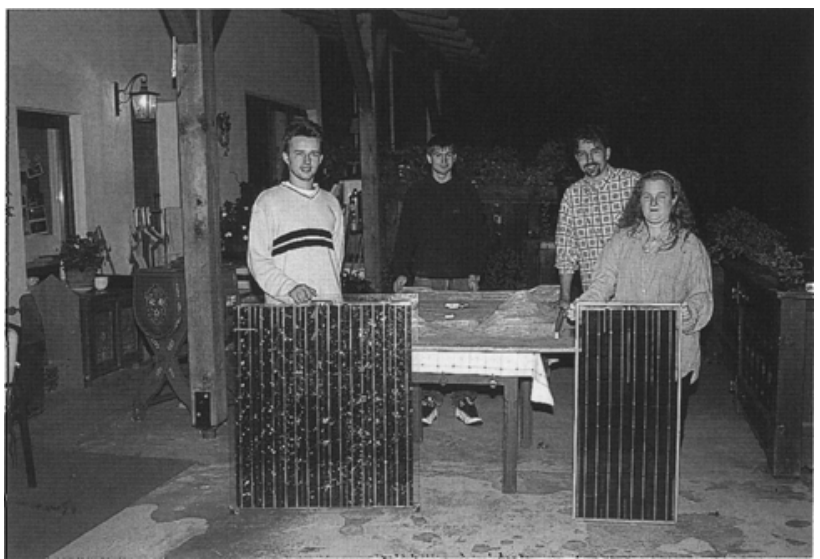

Abb. 2. Projekt Ladakh - Jugendgruppe. Das Sozialzentrum liegt in einer Wüste aus Stein und Sand in $3500 \mathrm{~m}$ Seehöhe

\section{Elektrische Energie für Nepal}

Nepal hat sehr entlegene Gebiete, die größtenteils nur zu Fuß erreicht werden können. Darum wurde ein äußerst kompaktes Kleinwasserkraftwerk, fix und fertig in einem Container installiert, entwickelt. Das KWKW gibt es in Größenordnungen von 12,6 kVA bis $360 \mathrm{kVA}$ und wird durch eine speziellen Konstruktion senkrecht eingebaut, um Platz zu sparen. Die Containeranlage kann in drei für den Hubschraubertransport taugliche Größen geteilt werden. Damit ist das KWKW auch in der Himalayaregion an jeder geologisch geeigneten Stelle aufstellbar (Abb. 3).

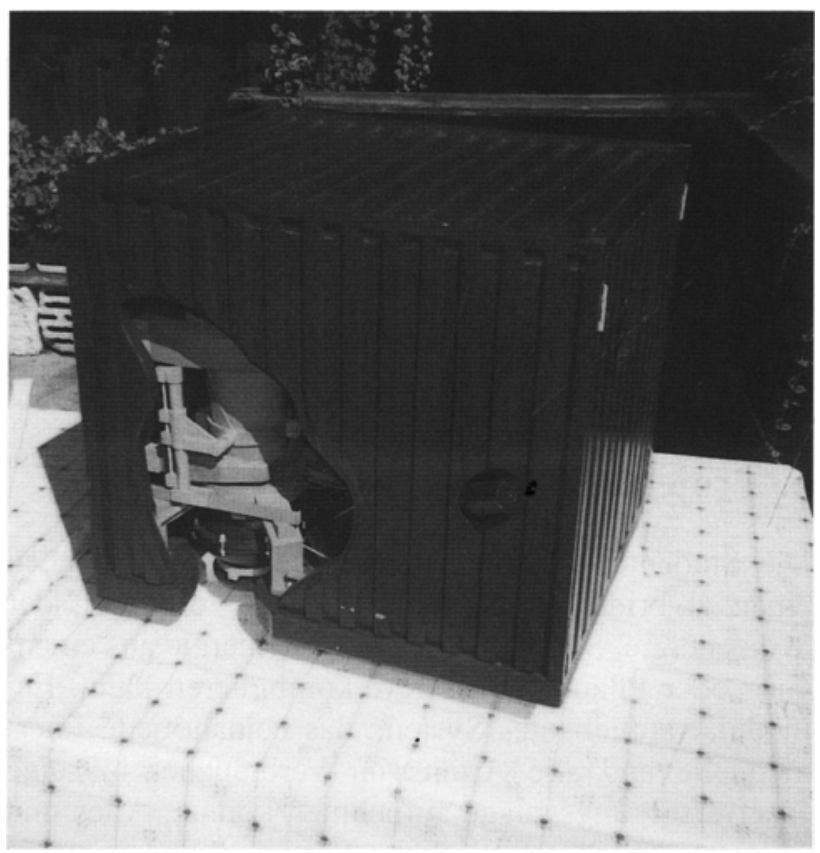

Abb. 3. Projekt Energie für Nepal. Kompaktwasserkraftwerk im Container und Gebirgsrelief vom Einsatzgebiet

Die photovoltaische Unterstützung hat mehrschichtige Aufgaben im Bereich Datentransfer zum Hauptkraftwerk und die automatische Anlagenüberwachung auch in Ausfallszeiten. Zudem kann Photovoltaik die Umrichter ganz vorzüglich unterstützen.

Die Projektgruppe erhielt den Youth Award für ihren enormen Einsatz um die Verbesserung der Lebensbedingungen in entlegenen Gebieten Nepals. Dank ihrer Leistungen wird in näherer Zukunft eine Grundversorgung an elektrischer Energie vorhanden sein.

\section{Projektarbeiten von Vorarlberger Schulen}

Die Aufgabenstellung für die Schüler einer Hauptschule in Bregenz war die Gestaltung einer PV-Anlage für ein Schulgebäude in der Größenordnung zwischen $1 \mathrm{kWp}$ und $3 \mathrm{kWp}$. 
Das Projekt „Schattenspender“, ein Sitzplatz mit Solardach, war für den Schulhof bestimmt.

Das Projekt ,5 vor 12“ mahnt: „es ist schon fast zu spät". Die Solarmodule als Stundenmarkierung zeigen uns aber bereits die Möglichkeiten für einen Ausweg.

Das Projekt „Sonnenspiegel“ sind Solarmodule, die sich in einem Biotop spiegeln. Das Projekt zeigt, daß "Sonne und Wasser zusammengehören“".

Das Projekt „Skateboard“" ist ein an die Schulwand gelehntes Skateboard, bei dem die Form durch Module erreicht wird, die zwischen Drahtseile gespannt sind.

„Das Buch“ soll uns zeigen, daß fast alles Wissen aus Büchern kommt. Die Seiten des Buchs sind aus Solarmodulen gemacht. In Büchern finden wir auch, wie Strom aus Sonne gewonnen wird.

Die Schüler aus Vorarlberg erhielten den Youth Award für Thre Bereitschaft zum Umdenken auf erneuerbare und umweltfreundliche Energieformen. Die Arbeiten stellen einen repräsentativen Querschnitt über das Denken, die Vorstellungen und die Kreativität junger Menschen dar.

\section{Projekt Solarcontainer}

Die Studenten der Gesamthochschule Kassel haben einen Hybridcontainer konzipiert und errichtet, der Windenergie, Sonnenstrom und die Energie aus einem Gas- oder Pflanzenöl-BHKW kombinieren kann. Ein modular aufgebautes System, das beinahe jede Energiequelle und alle Arten von Verbraucher in seine Inselversorgungsanlage aufnehmen könnte. Alles und noch zusätzlich erforderliche Batterien sind in einen handelsüblichen Container eingebaut.

Ein mit der Sonne gekoppelter Bierpreis lockte während der Pausen technikinteressierte Feinspitze aus den Vortragsräumen auf den Heldenplatz. Die Anlage hat viele Inselanlagenbetreiber, -betreuer und -errichter so begeistert, daß sie so mancher gerne gleich mitgenommen hätte. Der Container wird in Zukunft ein hervorragendes Schulungsmodell für Studenten in Kassel sein und eröffnet Möglichkeiten für weitere Entwicklungsarbeiten.

Die Studenten erhielten den Youth Award für die vorbildliche Realisierung einer Hybridanlage aus erneuer- baren Energien. Es zeigt in eindrucksvoller Weise, daß die Kombination von Photovoltaikmodulen und Windgeneratoren die Nutzung von erneuerbarer Energien erheblich verbessert.

\section{Schlußbemerkung}

Dr. Hofmann - Geschäftsführer der ASE Alzenau war von den Arbeiten so begeistert, daß er spontan ein Solarspielwerk an die Hauptschule Pfunds und einen Gutschein für eine 300-Wp-PV-Anlage als Starthilfe für das Projekt Ladakh überreichte.

Alle diese Projekte zeigen uns aber auch, wo Jugendliche eine Partnerschaft mit uns eingehen würden, wenn die Verantwortlichen aus Politik, Wirtschaft und Energiekonzernen sich ihrer Verantwortung für unsere künftigen Generationen klar würden. Standpunkte könnten sich annähern.

Auch die Finanzierung der Großprojekte im Bereich Wasserkraft verschlang in der Vergangenheit einen großen Teil des Geldes unserer Vorväter und trotzdem haben die Menschen damals das Kapital unserer heutigen Stromversorgung aufgebracht, um für die Nachkommen den Grundstock für eine gesicherte Energieversorgung zu schaffen. Sind nicht auch heute wir und die Unternehmen verantwortlich, daß in Zukunft die Energie gesichert ist? Man hat auch damals in eine neue Technik investiert, die umweltfreundlich war und ist das Risiko des Neuen eingegangen. Warum wagen wir moderne Menschen einen ähnlichen Schritt nicht und steigen in die erneuerbaren Energien und damit auch in die Photovoltaik ein? Sind wir vielleicht zu ängstlich geworden?

Der Youth Award wird auch bei zukünftigen Europäischen PV-Konferenzen und bei der 3. Weltkonferenz für Photovoltaik in Japan eine Fortsetzung finden. In Österreich hat sich der Bundesverband für Photovoltaik dazu bereit erklärt, diese Auszeichnung jährlich an junge Menschen zu verleihen. Bereits jetzt können sich die Jugendlichen, die mitmachen möchten und Ideen haben, bei der Bundeswirtschaftskammer - Bundesverband für Photovoltaik, z. Hd. Herrn Dr. Günter Mock, Wiedner Hauptstraße 63, A-1045 Wien melden. Wir glauben, daß dies eine erfreuliche Ergänzung zu den jährlich verliehenen österreichischen Solarpreisen der Eurosolar-Austria sein wird. 